\title{
Mujeres en Ciencia y Tecnología. Percepciones sobre el rol profesional de las ingenieras en Mar del Plata
}

\author{
Women in Science and Technology. Professional role perceptions of female \\ engineers located in Mar del Plata
}

\author{
Victoria Marquinez \\ Vickymarquinez@hotmail.com \\ Universidad Nacional de Mar del Plata, Argentina - \\ CONICET, Argentina
}

Recepción: 24 Julio 2020

Aprobación: 29 Octubre 2020

Publicación: 01 Marzo 2021

Cita sugerida: Marquinez, V. (2021). Mujeres en Ciencia y Tecnología. Percepciones sobre el rol profesional de las ingenieras en Mar del Plata. Descentrada, 5(1), e132.

https://doi.org/10.24215/25457284e132

\begin{abstract}
Resumen: Este artículo propone problematizar la baja participación femenina en el ámbito de la ingeniería. A partir de la realización de entrevistas en formato historias de vida, se pudo observar que, más allá de las barreras objetivas, existen aspectos subjetivos relacionados a las identidades de género que dificultan la participación femenina en este campo. Entre estos, se encuentra la necesidad de experimentar un proceso de adaptación por parte de las mujeres; el ejercicio de perfiles laborales relacionados a las habilidades consideradas femeninas; así como también la fuerte correlación entre las identidades profesionales y maternales.
\end{abstract}

Palabras clave: Ciencia y Tecnología, Ingeniería, Género.

\begin{abstract}
This article focuses on exploring the low level of female participation in the engineering field. Using a life-story methodology beyond objective barriers, it could be observed that there are subjective aspects regarding gender identities that difficulty the female participation in this field. Some of these subjective aspects are: the fact that women need to undergo an adaptation process; the development of labor profiles when considering female skills; and finally the strong relationship between the professional and maternal identities.
\end{abstract}

Keywords: Science and Technology, Engineering, Gender.

\section{INTRODUCCIÓN}

La situación de las mujeres en la ciencia y la tecnología es una problemática que ha adquirido reconocimiento en la agenda de la política mundial. Esto se debe a que, desde comienzos del siglo, se ha señalado la baja representación femenina en las carreras científicas, en especial en los campos de las ciencias exactas y naturales, y las ingenierías (Estébanez, 2003; 2004). Para disminuir la brecha de género, se han implementado diversas políticas públicas destinadas a la promoción de este tipo de disciplinas en el ámbito educativo; a programas de apoyo y premios a mujeres científicas; y políticas de igualdad de género en los sistemas nacionales de investigación (López-Bassols, Grazzi, Guillard y Salazar, 2018). En esta misma línea, diversos estudios se han concentrado en identificar las barreras estructurales que impiden la participación de las mujeres en el ámbito de la ciencia y la tecnología, destacando la discriminación de género y el tipo de socialización y educación que reciben las niñas. Para resolver esta situación, se propuso una transformación en los procesos de socialización y la implementación de políticas que fomenten la igualdad de oportunidades. 
Como afirma Judy Wajcman, estos estudios situaron el problema en las propias mujeres y no problematizaron cuestiones más amplias sobre la tecnociencia y sus instituciones. No se reconoce que las recomendaciones para la promoción de la igualdad de oportunidades, exigen a las mujeres que "sustituyan aspectos fundamentales de su identidad de género por una versión masculina de los mismos" (Wajcman, 2006, p. 27).

Para contribuir a estos debates, en este artículo propongo situar el análisis en aquellos aspectos del campo de la ciencia y la tecnología que se presentan como problemáticos para las mujeres. Partiendo puntualmente de las experiencias de las ingenieras que trabajan en Mar del Plata, indagaré en las trayectorias y en las identidades laborales construidas por mujeres que lograron insertarse en este ámbito. Siendo trabajadoras que se desempeñan en espacios masculinizados, el análisis de sus percepciones puede brindar aportes significativos para continuar problematizando las barreras que mantienen la segregación de género en el mercado laboral.

La situación particular de las ingenieras adquiere especial relevancia en la ciudad de Mar del Plata si se tiene en cuenta su importante desarrollo industrial, especialmente en el sector pesquero, en el textil y en la construcción. Actualmente, alberga una gran cantidad de industrias de diversas áreas y demandantes de profesionales de ingeniería. Además, desde 1970 la Universidad Nacional de Mar del Plata incluye en su oferta educativa la carrera de ingeniería. Habiendo ampliado las opciones en esta disciplina, la ciudad se constituye como polo educativo -atrayendo a estudiantes tanto intra e interprovinciales, como del exterior-, vinculado a su importancia en el mercado de trabajo regional.

Las reflexiones y conclusiones que se exponen a continuación, surgen del análisis de 25 entrevistas en formato historia de vida, distribuidas entre 15 ingenieras y 10 ingenieros residentes en la ciudad de Mar del Plata. ${ }^{1}$ Las edades de las y los entrevistados varían significativamente, abarcando una franja etaria que va desde los 27 a los 67 años. Esto me permitió comparar las distintas percepciones en torno al momento del curso de vida en que se encuentran. Además, las y los profesionales que se entrevistaron pertenecen a distintas ramas de la ingeniería. De esta manera, pude hacer foco en las diferencias que se generan entre las especialidades más masculinizadas y las que tienen mayor presencia femenina.

Para dar cuenta de las particularidades que adquieren las identidades profesionales en este sector, el artículo está organizado a partir de tres ejes de análisis. Primero, indago las motivaciones que movilizaron a estas mujeres a escoger una profesión considerada masculina, señalando también los obstáculos que se les presentaron en su inserción. Continuando con las trayectorias profesionales, presento las construcciones identitarias correspondientes a su ejercicio profesional y las características que toman en relación a las identidades de género. Por último, pensando al ámbito familiar y laboral como dos campos imbricados (Barrère-Maurisson, 1999), describo cómo se conjugan estas esferas en la trayectoria de las entrevistadas, haciendo hincapié en las lecturas que ellas mismas hacen sobre la maternidad y su valoración profesional. A partir del análisis de estos tres ejes, intentaré dar cuenta de los procesos subjetivos que atraviesan las mujeres que se insertan en espacios masculinizados y socialmente valorados, como es el caso de la ingeniería. Considerar este aspecto puede ser fundamental para el desarrollo de las políticas de género, enfocadas en mejorar la situación de las mujeres en la ciencia y la tecnología.

\section{INGENIERÍA Y MASCULINIDAD}

La ingeniería cumplió un rol fundamental en el proceso que identificó la tecnología como parte constitutiva de la masculinidad. A finales del siglo XIX, el saber científico y tecnológico se convirtió en una figura protagónica en el crecimiento industrial, especialmente en el desarrollo de la química, la electricidad y la mecánica (Rubio Mayoral, 2006). En las innovaciones tecnológicas que dieron paso a la Segunda Revolución Industrial, los ingenieros ocuparon un papel privilegiado, definiendo lo que se consideraba o no tecnología, al mismo tiempo que se consolidó esta profesión como un saber de varones blancos de clase media. De esta manera, las "máquinas masculinas" se convirtieron en la tecnología moderna, dejando fuera 
de estos límites todos aquellos artefactos e ingenios femeninos (Ruth Oldenziel, citada por Wajcman, 2006). Es así, como también quedó olvidado el papel de las mujeres en el origen de los primeros desarrollos tecnológicos, particularmente el de las mujeres indígenas, a las cuales se las puede considerar como las primeras tecnólogas debido a su función como cosechadoras, procesadoras y almacenistas. La figura del ingeniero se identificó con la imagen de la masculinidad, en la cual el saber técnico, la productividad, la racionalidad, el liderazgo y la condición de proveedores juegan un papel central. En palabras de Judy Wajcman, "el discurso de la masculinidad se movilizó para garantizar que se erigieran fronteras de clase, raza y género en torno al bastión de la ingeniería" (Wajcman, 2006, p. 30).

Las consecuencias de la relación tecnología-masculinidad están a la vista. Desde que las mujeres ingresaron a la Universidad, en el ámbito de la ciencia y la tecnología a nivel general, y específicamente dentro de la ingeniería, tuvieron poca participación. Así, entre 1921 y 1940 las egresadas en Ingeniería de la Universidad de Buenos Aires no alcanzaron a representar el 1\%, mientras que en la Facultad de Filosofía y Letras aumentaron a un rango de entre 12 y 18\%, escalando al 30 y 38\% en Medicina. Hacia la década de 1960, cuando la participación femenina en la universidad aumentó considerablemente, las estudiantes de Filosofía y Letras representaban el 70\% en 1964, disminuyendo al 32\% en las Ciencias Médicas y a menos del 20\% en Ingeniería (Lorenzo, 2016). Actualmente, las mujeres siguen aumentando su participación como nuevas inscriptas en las carreras de grado, alcanzando el $62 \%$ en el 2016. Aunque este aumento se vio reflejado en la carrera de Ingeniería, en donde las ingresantes pasaron de representar el 22\% en 2012 al 24\% en el 2016, el porcentaje se mantiene bajo, especialmente si se compara con carreras como Enfermería que representaron para ese mismo año el $80 \%$ de nuevos/as inscriptos/as, Letras (74\%) y Medicina $(67,75 \%) .{ }^{2}$ Con respecto a la estructura científica, de acuerdo a la Red Iberoamericana de Indicadores de Ciencia y Tecnología (RICYT), en Argentina, la participación de las mujeres en la investigación alcanza el 53\%. Sin embargo, al examinar los sectores y tipos de actividad, la aparente paridad desaparece. Es así, como ellas sólo representan el $27 \%$ de quienes investigan en el sector privado. En parte, esto se debe a que los principales campos de I+D en el ámbito empresarial, como las disciplinas tecnológicas y las ingenierías, mantienen una baja tasa de participación femenina (López-Bassols, Grazzi, Guillard y Salazar, 2018).

Por lo tanto, las barreras de género que se construyeron alrededor de la ingeniería se mantienen. Esto también se percibe en los discursos de las/os entrevistadas/os, ya que se manifiesta la fuerte identificación de la ingeniería como un territorio masculino. En las lecturas que ellas/os mismas/os hacen sobre las razones que las/os llevaron a elegir esta profesión, como figuras motivadoras solo se mencionan identidades masculinas. De esta manera, se alude al padre, e incluso a personajes ficticios como "McGyver", ${ }^{3}$ para referirse a la persona que las/os introdujo al mundo de la técnica y al cual deseaban parecerse como profesionales. Al mismo tiempo, desde una posición opuesta, se evocan las figuras femeninas para atribuirles un sentido negativo. Solo se las nombra en su rol tradicional de ama de casa, representada como lo no deseado, aunque la mayoría de estas mujeres se responsabiliza y realiza la mayor parte de las tareas domésticas y de cuidados en sus propios hogares.

La primera reacción mía es quiero ser como mi papá (Graciela, 36 años, ingeniera ambiental).

Yo no quería quedarme solamente con una imagen de una ama de casa o algo así, y siempre me gustó el armar, desarmar, el poder innovar, el poder buscar soluciones (Belén, 40 años, ingeniera en sistemas).

$\mathrm{Al}$ reflexionar sobre las figuras motivacionales, los roles de género se mantienen. La permanencia de estos estereotipos afianza la clasificación y caracterización de ciertos puestos y ocupaciones como propios de cada género. Se definen así "territorios masculinos y femeninos en el mundo del trabajo". Esto corresponde a una organización binaria de la sociedad, la cual también se manifiesta en la construcción de la ingeniería. En esta, es fundamental la dicotomía entre ciencia y sensualidad, entre lo duro y lo blando, entre las cosas y las personas. Los sistemas binarios se sitúan dentro de redes de símbolos generales que cumplen una importante función en la construcción de la "inferioridad de las mujeres", ya que, siguiendo con la misma lógica, al mismo tiempo que se las identifica con la naturaleza y la emoción, se las opone a la razón (Arango, 2006; Keller, 
2001). La dicotomía entre naturaleza/cultura justifica y perpetúa la desigualdad social, legal y política de las mujeres, asimilando un argumento determinista que justifica la "distribución de roles sociales y el escaso posicionamiento de las mujeres en ciencia y tecnología” (Munévar, 2011, p. 238). La fijación de las mujeres del lado de la "naturaleza", las opone al área tecnológica como una cuestión innata a la femineidad.

En este marco, el acceso de las mujeres a la ingeniería se dificulta doblemente. Por un lado, la socialización diferencial y estereotipada entre las niñas y niños, influye en la identidad -social e individual- de la persona, determinando las creencias y expectativas propias de acuerdo al género (Eccles y col., 1993, citado en Lisbona, Sáinz y López-Saéz, 2004). Por otro lado, el ámbito tecnológico se erige en torno a un modelo androcéntrico, que implica en muchos casos tener que adoptar comportamientos considerados tradicionalmente como masculinos. Por este motivo, a la mayoría de estas mujeres se las considera "trabajadoras extranjeras", es decir, no pertenecen al lugar donde se desempeñan. Es así, como no solo no se las incentiva para ejercer tareas relacionadas con la ciencia y la tecnología, sino que también, cuando eligen este campo de estudio, se ven obligadas a experimentar un "proceso de aculturación” (Panaia, 2013).

Este proceso es mencionado por las entrevistadas como una etapa de "adaptación”, en el cual se adquiere el vocabulario y las formas de actuar que se consideran indicadas para relacionarse en el ámbito masculino. Jill Conway, Susan Bourque y Joan Scott (1987) observan que, culturalmente, se producen formas apropiadas de comportamiento femenino y masculino, transmitidas a través de los usos de símbolos y del lenguaje. A partir de un conjunto de disposiciones, las sociedades transforman la diferencia sexual anátomo-fisiológica en distinciones sociales que marcan, entre otras cosas, la relación entre las personas (Rubin, 1986). El sistema sexo/género, que define Gayle Rubin, está fuertemente presente en la identidad de las entrevistadas. Así, al igual que una persona que emigra de su país, ellas sienten que debieron modificar sus normas culturales. Asimismo, aunque se adapten al mundo masculinizado, no son tratadas como pares iguales. Las formas de sociabilidad entre varones y mujeres mantienen, en la mayoría de los casos, una distancia marcada por la utilización formal del comportamiento y del lenguaje. Esto puede incidir en el hecho de que las relaciones más íntimas entre compañeras/os se suelen dar entre las personas del mismo género, lo que nos invita a suponer que transitar la carrera universitaria con compañeros de otro género puede constituir para algunas una dificultad. A modo ilustrativo, el relato de tres ingenieras que cursaron su carrera entre las décadas de 1970-1980; 1990-2000; y 2000-2010, respectivamente, dan cuenta de que, a pesar de haber transitado su trayectoria universitaria durante contextos sociales diferentes, la percepción sobre la necesidad de adaptarse se mantiene:

Era mucho más tímida, cuando entré en ingeniería. Ahí bueno, tuve que sobrevivir, así que, se me fue toda la timidez, terminé la facultad no siendo tímida, siendo una charlatana (...) me fui aggiornando a eso de, estar con hombres, estudiar con hombres (Patricia, 58 años, ingeniera civil).

Dos mujeres nada más en electromecánica (...) fui a un colegio secundario de mujeres, pero mi hermano mayor era varón y él iba al industrial, entonces en casa siempre había varones, chicos, entonces eh... me fue fácil el trato con los chicos (...) como que eran más cuidadosos porque había una mujer, entonces como que se re cuidaban de no decir nada, de portarse bien (Julieta, 39 años, ingeniera mecánica).

Desde segundo año siempre cursé solo con varones, me adapté re bien, me acostumbré bien. Igual en mi trabajo, en mi trabajo son todos varones y tampoco tengo problema. Son muy respetuosos en lo que es el ambiente naval, no tengo ningún tipo de inconveniente (Florencia, 27 años, ingeniera naval).

Aunque en el discurso de las entrevistadas parece que esta transición finaliza en la adquisición de las "normas de sociabilidad masculinas", el resto de su trayectoria profesional nos señala que esta etapa se extiende. Para que las mujeres sean aceptadas en el ámbito de la ingeniería, también deben demostrar su capacidad y muchas veces se les exige más que a sus compañeros. Escenarios en donde se cuestiona la presencia femenina, tanto por parte de profesores en instituciones educativas -universidad o escuelas técnicas- como por superiores en la esfera laboral, son mencionados por las entrevistadas de todas las edades. Ante esta situación, las mujeres deciden acrecentar su esfuerzo para ser aceptadas, intentando muchas veces distinguirse de otras mujeres. El acceso al campo de la ingeniería significa para las profesionales el distanciamiento de lo 
considerado tradicionalmente como femenino, lo que mantiene estable la masculinización del territorio. De esta manera, no se problematiza el diseño y los valores que rigen esta profesión, como el individualismo, la competencia, las jerarquías fijas o la búsqueda de hegemonía y solo se limita a disminuir las barreras en el acceso a estos lugares (Rietti y Maffia, 2005).

El profesor nos dijo que... se dirigió a las mujeres especialmente y nos dijo que nos apuráramos a conseguir el novio que habíamos venido a buscar, porque la facultad de ingeniería no tenía lugar para 'calientasillas' (...) tocaron varios [profesores] que, si tenías todo perfecto y eras mujer, llegabas al 8, para tener el 10 tenías que ser varón (Susana, 49 años, ingeniera química).

Primera clase, dice, 'bueno yo quiero que conozcan mi visión, para mí las mujeres, a la cocina' dijo, 'desde ya que van a tener conmigo un 2 todo el año, si se piensan que van a hacer, cada vez que pasen a la teoría, como una receta de cocina’ (...) Yo me lo puse como desafío (Romina, 43 años, ingeniera civil).

Un profesor me preguntó qué hacía estudiando ingeniería naval. Pero ya te digo, después de rendir el primer parcial, no me miró más con cara distinta, porque me iba igual que el resto de los chicos, no tenía nada que ver (Florencia, 27 años, ingeniera naval).

Frente a estos sucesos, en los cuales generalmente las entrevistadas se esforzaron en demostrar su capacidad, la relación con el varón que la juzgó suele mejorar, hasta el punto de convertirse en una figura relevante en su formación profesional. Situaciones como las relatadas, nos permiten suponer que, ante el cuestionamiento proveniente de una persona de mayor jerarquía, muchas mujeres hayan abandonado los estudios. La experiencia de Julieta, ingeniera mecánica de 39 años, nos ayuda a imaginar esta situación. Notándose insegura con respecto a la carrera que había elegido -ingeniería electrónica-, decidió abandonarla durante algunos años al ser interpelada por un docente:

Lo que me hizo definitivamente dejar electrónica fue un profesor de 'Circuitos 1', un tal Toni. Un día le voy a preguntar una cosa. Ya en clases anteriores, había hecho un comentario de que había muchas, que cada vez había más mujeres en la carrera, y que... no entendía por qué, porque la verdad que no era un trabajo para mujeres, la ingeniería no era algo de mujeres. En un momento, le voy a preguntar alguna cosa y me dice 'vos no sé qué estás haciendo acá, vos tenés que estudiar maestra jardinera' (Julieta, 39 años, ingeniera mecánica).

Estos fragmentos nos muestran que, si bien en los últimos años la cantidad de mujeres que eligen esta profesión ha aumentado, los cuestionamientos por la idoneidad femenina en la técnica persisten. Casi la totalidad de las entrevistadas más grandes -quienes cursaron su carrera entre las décadas de 1970 y 1980narran hechos discriminatorios con sus profesores, sin distinción de especialización. Esta última variable adquiere mayor importancia entre las mujeres de mediana edad y las más jóvenes, ya que las que relatan alguna actitud discriminatoria son mujeres que eligieron áreas más masculinizadas, como mecánica, civil o naval. Aquellas que siguieron ramas en las que se acrecentó la tasa de participación femenina, como alimentos y química, no reconocen ningún tipo de discriminación. Aunque, si tenemos en cuenta que las situaciones que las entrevistadas mencionan son momentos en que la violencia se presentó de manera explícita, podemos pensar que existen otros comportamientos de violencia "naturalizada" y que no son reconocidos por ellas. ${ }^{4}$

La segregación laboral dentro de la ingeniería se manifiesta de dos formas. La primera corresponde a la elección de la especialización. Ello se debe a que, mientras que en las áreas de alimentos, ambiental y química, constituyen a nivel nacional el 73\%, 63\% y 51\% de las graduadas del 2017, en ingeniería nuclear no egresaron mujeres ese año, y en las ramas de electromecánica, electrónica y mecánica, representaron entre el 3\% y el 5\%. ${ }^{5}$ Estos números nos señalan que las mujeres suelen optar por aquellos tipos de ingeniería que no se encuentran fuertemente asociados a la tecnología dura, sino que eligen las especialidades que son afines al campo de saberes considerados femeninos. Lo mismo ocurre en el desarrollo profesional -y esta es la segunda forma en la que se visibiliza la segregación- ya que las mujeres se agrupan en los sectores del mercado laboral más alejados a este tipo de tecnología.

Esto último sucede, entre otras cosas, porque al igual que otras trabajadoras, suelen privilegiar el horario, el salario estable y la flexibilidad. Es así como la mayoría de estas mujeres se concentran en los empleos del sector 
público, los cuales, además de poseer mecanismos formales de promoción laboral, facilitan la conciliación con las responsabilidades de cuidado. En Mar del Plata, los varones duplican en número a las trabajadoras asalariadas del sector privado. No sucede lo mismo en el empleo público, donde se alcanza una aparente paridad. En consonancia con esta situación, la mayoría de las entrevistadas se desempeñan como empleadas en la Municipalidad o en la Universidad como docentes e investigadoras. Las palabras de Graciela, ingeniera ambiental y madre de dos hijas/os, reflejan la dicotomía que se suele presentar en estas profesionales entre tener un trabajo de alto prestigio y remuneración, o mantener un puesto laboral que les permita cumplir satisfactoriamente con sus responsabilidades familiares. Ella rechazó otros trabajos con sueldos más altos, por los beneficios que su puesto municipal como ingeniera le brindaba:

En la municipalidad al principio no ganás nada, pero... yo trabajaba de 8 a 3 de la tarde, y en el verano de 7 a 2 . Entonces, ya me iba y venía con los nenes, tenés muchas vacaciones. Para una mujer la municipalidad es genial, si no tenés un cargo importante (...) ni jefe, ni nada... porque estás como loco. Yo lo veo a mi jefe que le suenan 3 teléfonos a la vez, no, yo esa locura en mi vida no la quiero, no, no. Si uno lo piensa por el tema monetario, obviamente es un abismo lo que hay entre lo que cobra un ingeniero que lo que cobra un director (Graciela, 36 años, ingeniera ambiental).

Por otro lado, la falta de figuras femeninas en estos espacios también tiene fuertes consecuencias para la proyección profesional de estas mujeres. Alicia Palermo (2012) afirma que la elección de una carrera no tradicional provoca inseguridad sobre su futuro profesional. Esto se debe a la incertidumbre que mantienen sobre el logro de objetivos profesionales, dificultándoles anticipar la obtención de resultados y valorar los objetivos deseados. Las expectativas entre las estudiantes disminuyen, siendo común que las mujeres en la etapa universitaria no logren imaginarse como trabajadoras. La incertidumbre sobre su futuro profesional puede estar fundada también en que los estudios secundarios y/o puestos laborales ocupados por ellas durante su trayectoria universitaria, generalmente no estaban relacionados con su área de estudio. En cambio, los varones ya estaban vinculados con la carrera, sea por estudios secundarios o por trabajos relacionados. Así, de los/as 25 entrevistados/as, 9 realizaron sus estudios secundarios en una escuela técnica, distribuidos en 6 varones y 3 mujeres. Por otro lado, de los 10 entrevistados varones, 9 tenían trabajo antes de recibirse en un puesto relacionado a su profesión, mientras que solo 3 de las 15 entrevistadas se encontraba en la misma situación. ${ }^{6}$

A lo largo de este apartado, se intentó dar cuenta de la dificultad que supone para el imaginario social desarticular la ingeniería como un territorio naturalmente masculino. Esto se debe a que, desde sus orígenes, la identidad del ingeniero se construyó sobre una lógica sociosexuada y estableció delimitaciones con otras áreas ocupacionales de menor valoración social. Si se mantiene la identificación entre ingeniería -y tecnologíay masculinidad, podemos suponer que la causa de la subrepresentación femenina en esta área, no se debe únicamente a una socialización diferenciada en la infancia. La tecnología está inserta en una cultura de la masculinidad (Wajcman, 2006) y, para insertarse, las mujeres deben renunciar a una parte de su propia identidad de género. Entonces, ¿cómo afecta el proceso de adaptación a la percepción de su propia identidad?, ¿cómo se inscribe el género en la construcción del rol profesional?

\section{Mujeres ENTRE VARONES}

Para comprender la construcción de las identidades de las ingenieras, es importante dar cuenta de que el género constituye una identidad social que implica vivir y actuar bajo un conjunto de representaciones. Siguiendo a Nancy Fraser, las identidades sociales "se construyen discursivamente en contextos sociales históricamente específicos, son complejas y plurales; y cambian con el tiempo" (Fraser, 2015, p. 171). $\mathrm{Al}$ definirlas como "plurales", señala las diversas prácticas que la constituyen. De esta manera, nadie es simplemente mujer, sino que también debe considerarse su clase, educación, profesión, religión, ideología, etc. Para analizar con mayor precisión las diferentes realidades que viven las mujeres, es fundamental incluir 
el concepto de "interseccionalidad" (Crenshaw, 1995). Éste refiere a la situación en la cual las desigualdades se profundizan cuando entran en relación distintos elementos -como el género, la etnia o la clase social-que se interseccionan de forma diferente en cada caso y grupo social, y muestran las estructuras de poder existentes (Expósito Molina, 2012). Las entrevistadas están atravesadas por las problemáticas típicas de personas que constituyen parte de la clase media profesional y, particularmente, por haber elegido como campo profesional un espacio socialmente valorizado, con tasas de masculinidad muy superiores a las femeninas.

La masculinización de la ingeniería incide en las formas en que se configuran las identidades y percepciones. Entre las entrevistadas, el género se utiliza para valorizar el trabajo de las ingenieras. Mientras que, en los espacios más feminizados del campo como la investigación en la rama de la química o alimentos, se encuentran casos en los cuales se les asigna mayor capacidad intelectual a las mujeres, en detrimento de los varones; en los ámbitos masculinizados, se destaca el género femenino para reforzar la percepción positiva de su trayectoria con respecto a sus pares masculinos. En el último caso, haber conseguido desarrollar una carrera profesional en un contexto difícil para las mujeres, es un factor clave para la valorización. Las lecturas de la propia historia de vida en las cuales se expresa esta connotación valorativa, suelen pertenecer a las mujeres que se encuentran en los últimos pasos de su trayectoria, próximas a jubilarse. Patricia, ingeniera civil, trae a colación la opinión de un miembro del sindicato al cual pertenece para demostrar la aceptación que tiene en el ámbito laboral. Tal como ella lo recuerda, en el discurso de esta persona está latente la idea de que ella constituye una excepción respecto de las mujeres. Es decir, al mismo tiempo que resalta sus logros, manifiesta su creencia de que estas habilidades son ajenas al género femenino.

Alguien del sindicato me dijo 'Patricia, es increíble, vos siendo mujer, con tantos hombres a cargo y gente mayor, donde voy me hablan bien de vos' dice, 'y eso no cualquiera podría decirlo, y más siendo mujer' (Patricia, 58 años, ingeniera civil).

En la percepción de sus roles laborales también se valoriza, por parte de las entrevistadas, la implementación de las prácticas consideradas femeninas en la realización de tareas calificadas tradicionalmente como masculinas. Es interesante observar de qué manera se construye una identidad propiamente femenina en los modos de ejercer el mando, relacionados al ejercicio de la comprensión y la empatía en oposición al autoritarismo. Por el contrario, los varones entrevistados se suelen identificar en mayor medida con este último perfil. Si bien entre algunos ingenieros también aparece como parte de su función el escuchar al otro/a e incentivar la participación de las personas que dirigen, muchos mantienen características como la formalidad y el autoritarismo.

\footnotetext{
Mucho personal masculino y gente mucho más grande que yo, pero me iba bien, por la manera que tengo yo de... de tratar de motivar un equipo, de que vamos por un objetivo, digamos, pedir las cosas bien. Tomarte la autoridad, pero hacer que te respeten, no por tu puesto si no porque vos los respetás a ellos y los comprendés a ellos (Cecilia, 36 años, ingeniera en alimentos).

El puerto tiene una clase social bastante marcada y diferenciada, lo cual, es como hablamos nosotros, normalmente andan a los garrotazos, si no los garroteas no andan, pero si los garroteas no sos un déspota, no les molesta, en el buen sentido de la palabra ¿no? necesitan que vos estés todo el día con el garrote, machacándole para que funcionen (Gastón, 45 años, ingeniero mecánico).
}

En este sentido, existen numerosos estudios que sostienen la idea de que se conforma un tipo de liderazgo femenino en oposición al masculino tradicional (Loden, 1986; Kaufmann, 1996) que se caracteriza por un control reducido, comprensión, colaboración y niveles altos de rendimiento (Barberá, Ramos y Sarrió, 2003). Estos estilos de liderazgo no responden a un carácter ontológico, sino que son producto de la socialización diferenciada que organiza a los géneros de manera dicotómica. Así, las mujeres son motivadas a formarse como seres sensibles, emotivos y cooperativos, mientras que los varones son incentivados para desarrollar aptitudes de autoridad, control, eficacia, agresividad, independencia y racionalidad (García Prince, 2008). Sin embargo, no hay un fuerte consenso sobre esta tajante división. Es así como otros estudios sostienen que las mujeres se adaptan a un modelo androcéntrico de profesional para llevar a cabo puestos de liderazgo (Cuadrado, 2003; 
Heller, 2011). Entre las entrevistadas, se encuentran ejemplos de ambos casos. Dentro del último perfil se ubica una de ellas, quien afirma adoptar una actitud desde el control y la autoridad, mientras que manifiesta su dificultad en llevar a cabo tareas en equipo. En el lado opuesto, otra ingeniera manifiesta no tener el perfil de líder por la falta de las características propias relacionadas al modelo de profesional androcéntrico. Por este motivo, no aspira cargos jerárquicos. Es significativo tener en cuenta que asume esta incapacidad de liderar, luego de postularse a distintos puestos directivos dentro de la empresa donde trabaja, que no consiguió.

Entre los múltiples factores que conforman el "techo de cristal", Mabel Burín (1996) menciona la permanencia de los estereotipos de género. Esto implica, por una parte, sufrir mayores exigencias que la de sus pares varones; y por otra, la internalización de este ideal femenino. Las mujeres mismas creen y asimilan como elecciones propias, las creencias que circulan dentro del ambiente laboral que las coloca en una posición de desventaja. Representaciones que sostienen que "a las mujeres no les interesa ocupar puestos de responsabilidad", o que "no pueden afrontar situaciones difíciles que requieren actitudes de autoridad y poder", se mantienen y dificultan su acceso a puestos jerárquicos.

Por lo tanto, las distinciones de género juegan un papel importante en la construcción de identidades laborales. Además del efecto que tienen en la elección diferenciada en las carreras universitarias, también influyen en las formas de liderazgo que construyen las entrevistadas y los roles que adquieren dentro de la organización laboral. En este último sentido, es significativo el caso de Florencia quien afirma haber obtenido su actual trabajo por su género femenino. En 2012, formó parte del primer grupo de graduados/as de ingeniería naval de la UTN de Mar del Plata y, como consecuencia, la mayor parte de ellos/as iniciaron su búsqueda laboral en simultáneo. En parte, esto explica por qué ella define a este contexto como un momento difícil para insertarse en su profesión. A diferencia de algunos de sus compañeros, rápidamente comenzó a desempeñarse en uno de los astilleros más importantes de la ciudad. El puesto lo consiguió, como ella misma afirma, debido a que la presidenta del astillero necesitaba el perfil de alguien que le diera un orden a la documentación. Aunque algunas de sus tareas sí se relacionaban a su profesión -de hecho, pudo desarrollarse como ingeniera dentro de la empresa- esto señala que las cualidades asociadas culturalmente a las mujeres persisten y, muchas veces, se trasladan a sus posibilidades laborales. El hecho de que haya sido empleada por otra mujer, quien no solo ejerció su profesión en un espacio masculinizado, sino que supo romper - ¿de qué manera?- ${ }^{8}$ el techo de cristal y obtener la dirección de esa empresa, no garantiza que se desarmen los estereotipos de género. Entonces, se mantiene el orden de este espacio y son ellas quienes deben adaptarse y demostrar su capacidad. Sólo después, podrán comenzar a ser consideradas como pares iguales.

Desempeñarse laboralmente en un ámbito de baja participación femenina tiene diversas consecuencias para ellas. Además de las limitadas y débiles relaciones laborales que puede implicar para algunas mujeres el desarrollarse en contextos típicamente masculinos, en general, su situación es más difícil que la de los varones cuando están en minoría (Fueguen y Biernat, 2002, citado en Lisbona, Sáinz y López-Saéz, 2004). Si bien muchas hacen hincapié en la satisfacción de sus pares con su ejercicio laboral, habiendo sido felicitadas por estos/as, también se reconoce una mayor exigencia de los varones para con ellas. Sienten que deben demostrar constantemente su capacidad:

Una como mujer y profesional tiene que demostrar dos veces su capacidad, es como que tenés que demostrar siempre que nunca te regalaron el título (...) como ingeniera es como, algo muy machista (Belén, 40 años, ingeniera en sistemas).

Cómo llegué a la dirección [del departamento de ingeniería eléctrica de la UNMDP], te interesará saber. Es un logro bastante importante, porque en un núcleo tan machista y de hombres, fue un gran logro. Me costó un poco (...) fue duro porque yo también me sentí como que ahí había un componente medio machista en algunos docentes que ponían trabas. Que en definitiva después se zanjaron rápidamente, porque cuando yo empecé a trabajar bien y se dieron los resultados rápidos (Ana, 63 años, ingeniera eléctrica).

$\mathrm{Al}$ igual que otras entrevistadas, Belén y Ana coinciden en caracterizar el ambiente de la ingeniería como machista. Aunque sí reconocen el malestar provocado por el trato diferencial de sus pares masculinos, se reitera y valoriza en los discursos el esfuerzo realizado para demostrar su habilidad. Nuevamente, el acento 
se sitúa en su propio accionar. Es decir, son ellas quienes deben seguir esforzándose para ser aceptadas. Aunque las ingenieras sufran un proceso de adaptación al insertarse en el mundo de la ingeniería, a lo largo de su carrera profesional deben demostrar continuamente su pertenencia al campo. Como consecuencia, el género femenino se transforma para estas mujeres en un factor de reconocimiento y actúa como valorizador, no solo de su propia trayectoria, sino que también se resalta la capacidad de otras ingenieras en las cuales, posiblemente, se ven reflejadas. Por esto y por la permanencia de estereotipos femeninos que tienen fuertes injerencias en su trayectoria, se puede afirmar que el género atraviesa constantemente su propia percepción como profesionales. Ahora bien, ¿cómo interviene la maternidad en las apreciaciones profesionales?, ¿cómo se conjugan los deseos y responsabilidades familiares con la percepción del rol profesional?

\section{Profesión y maternidad}

Históricamente, el trabajo de las mujeres ha sido comprendido como complementario al de los varones, atribuyéndoles la maternidad como rol primordial en la vida. Esto implicó la despreocupación de su condición laboral y su participación en el mercado como mano de obra más barata. Tal situación responde a una división sexual del trabajo, que funciona tanto en la estructura laboral como familiar. En este reparto, varones y mujeres ocupan lugares específicos "dentro de la familia que se remite a aquel que posee el empleo, y recíprocamente" (Barrère-Maurisson, 1999, p. 148).

Actualmente, aunque la mitad de las mujeres argentinas -en edades entre 14 y 64 años y que viven en zonas urbanas- participan en el mercado laboral, ${ }^{9}$ numerosos estudios dan cuenta de que el crecimiento de la Población Económicamente Activa (PEA) femenina, no fue acompañada de un aumento de las responsabilidades domésticas y de cuidados de los varones (Wainerman, 2007). Esto supuso que las mujeres debieran cumplir una "doble jornada de trabajo" (Hochschild y Machung, 1989), insertándose en el mercado laboral sin abandonar la organización y el ejercicio de las tareas domésticas, y de cuidado no remuneradas. Según la última encuesta sobre Trabajo No Remunerado y Uso del Tiempo del tercer trimestre del 2013, la tasa de participación femenina en las tareas domésticas es del 88,9\%, mientras que en los varones baja al $57,9 \%$. El mantenimiento de ambas responsabilidades implica mayores dificultades para la participación en el mercado de trabajo, sobre todo cuando en los hogares hay infantes, debido a que el mayor o menor número de hijas e hijos dificulta la compatibilización de la esfera familiar y laboral (Wainerman, 2007; Actis de Pasquale y Lanari, 2015; Cutuli y Aspiazu, 2015).

Las tensiones entre el campo productivo y el reproductivo, intervienen en la construcción de la identidad profesional. Las identidades genérico-profesionales se sostienen en dos ejes contradictorios: la maternidad y la profesión. Para conciliar ambos roles, se ejercen diversas estrategias: muchas mujeres le otorgan preferencia a la maternidad y otras a la profesión, sin dejar de lado ninguno de los otros dos campos. Algunas, deciden no ejercer una estrategia de conciliación y optan por prescindir de una de las dos obligaciones. Ante esta situación, Guadarrama Olivera sostiene que las identidades laborales femeninas sufren "procesos contradictorios más generales de resignificación de las categorías genéricas socialmente configuradas y transmitidas, y las propias experiencias biográficas relacionales de las mujeres a partir de las cuales reconfiguran genéricamente los saberes aprendidos" (Guadarrama Olivera, 2008, p. 332). Las identidades profesionales están sujetas a las trasformaciones producidas dentro de la esfera laboral y familiar -así como también influyen en el desarrollo de estos ámbitos- y a los estereotipos profesionales, que incluyen los saberes y habilidades orientados genéricamente.

En las valoraciones de las entrevistadas sobre su rol profesional, se hace presente la articulación permanente de estas dos esferas como "ejes fundamentales e indisolublemente entrecruzados de sus identidades" (Díaz, Godoy y Stecher, 2007, p. 93). Es así como la importancia que adjudican al trabajo remunerado como uno de los espacios fundamentales de sentido, acción y responsabilidad, no reemplaza para ellas ni se opone al ámbito familiar. Asimismo, esta articulación se presenta con dificultades, especialmente con la llegada del/la primer/ 
a hijo/a. En muchas de las entrevistadas se percibe este hecho como un "cambio de prioridades", relacionado generalmente a la relación trabajo-familia. Frente a las transformaciones que se producen al momento de ser madres, la percepción sobre la combinación del rol maternal y laboral toma distintas formas. En algunos casos, la esfera familiar se posiciona por sobre la profesional, por lo que la percepción sobre su trabajo se desvaloriza y pasa a clasificarse como un mero medio para vivir. El caso más claro aparece en Julieta, ingeniera mecánica, quien se definía como "trabajo-dependiente". Luego de tener su primer hijo la valoración con respecto al trabajo se invirtió totalmente, hasta el punto en que afirma que "no quería volver".

Esto responde a una fuerte correlación entre sus identidades maternales y la percepción que tienen sobre su trabajo. En aquellas que defienden los roles de género tradicionales y mantienen un ideal de la maternidad intensiva, la importancia del trabajo como realización personal se debilita, afirmándolo en su fin meramente material. En cambio, entre las mujeres que expresan mayor independencia de sus roles familiares -sin desestimar la carga de responsabilidades domésticas y de cuidados- su profesión es percibida como una fuente de gran satisfacción, justificando en muchas ocasiones, su desarrollo profesional como constituidor de ejemplo a seguir para sus hijas/os. La mayor valorización del trabajo, para estas profesionales, parece haber tenido consecuencias en su desarrollo familiar. Entre estas, las entrevistadas más grandes se divorciaron y otras -de mediana edad- no tienen pareja ni hijas/os. Las más jóvenes, quienes también tienen una percepción positiva de su rol profesional, cuando planean su futuro familiar expresan su deseo de ser madres, aunque eso signifique para ellas sacrificar una parte de su ejercicio laboral.

Entre los varones, también se encuentran aquellos que perciben su trabajo como solo un medio para vivir, y en su relato -en comparación con los otros entrevistados- parecen participar más en el cuidado de sus hijas/os. Sin embargo, esta correlación no influye de manera objetiva en su desarrollo profesional, como sí lo hace en las trayectorias femeninas. Para ellos, las extensas jornadas laborales no son percibidas como una dificultad en la conformación de una familia. Los ingenieros de mayor y de mediana edad, han tenido entre 2 y 3 hijas/os, al mismo tiempo que recaía sobre ellos una gran responsabilidad laboral. Mientras que los más jóvenes, quienes no son padres, no proyectan una disminución laboral como consecuencia de sus responsabilidades paternas.

El trabajo productivo sigue constituyéndose como referente fundamental de la masculinidad (Díaz, Godoy y Stecher, 2007). Esto se manifiesta en la percepción de los entrevistados del trabajo como destino ineludible. Al mismo tiempo que se acepta incuestionablemente el trabajo remunerado de la mujer, se naturaliza el hecho de que sean ellas quienes ajusten su horario y condiciones laborales para equilibrarlo con sus responsabilidades de cuidado y domésticas. De esta manera, o se renuncia o rechaza determinado puesto laboral, o se produce una postergación de la maternidad durante el desarrollo más intensivo de la profesión. Andrea y Cecilia, ingenieras en alimentos e investigadoras del Conicet, optaron por esta última alternativa. Ambas postergaron su maternidad hasta el término de su doctorado ya que, tal como manifestaron, luego no tendrían tantas dificultades en conciliar su trabajo con el cuidado de un/a menor. A la hora de proyectar una familia, los puestos laborales o los tipos de trabajo se problematizan. Las creencias apoyan la idea de que los trabajos no competitivos -aquellos en los que no se debe garantizar disponibilidad full time y no se responsabilizan por otras personas a su cargo- se amoldan mejor al rol maternal y fortalecen la segregación laboral.

En la mirada de las entrevistadas que son madres, la relación no se percibe como complicada si se escogen determinados trabajos. Sin embargo, la trayectoria laboral de estas mujeres suele modificarse cuando los/ as hijos/as crecen. Así es como Graciela, por un lado, sostiene que la familia no perjudica el desarrollo profesional: "Si a vos no te deja desarrollarte profesionalmente tu familia, algo estás haciendo mal, porque tu familia no es un escollo, al revés, es el sostén para poder hacer”. Pero, por otro lado, aprovechando que sus hijas/os son mayores, planea nuevos proyectos profesionales. Entonces, aún en aquellas que manifiestan que la adaptación entre trabajo y familia no se dificultó, si vamos a los hechos, observamos que su percepción no se relaciona con los sucesos porque su desarrollo laboral sí se vio modificado por los ciclos vitales de sus hijas/os. La apreciación de Graciela, asimismo, señala que en ese período no hubo para ella conflicto en la conciliación. Al mismo tiempo que se insertó en el mercado laboral, ejerció un rol maternal que se acercaba a 
su ideal porque estaba acompañando a sus hijas/os la mayor parte del día. Esta tensión se nota en el relato de Belén, ingeniera en sistemas y madre de dos hijas/os, quien decidió renunciar a un puesto laboral altamente competitivo en una empresa internacional para incrementar su presencia en su hogar.

Yo no era feliz estando 15 días en un país extranjero cuando mi hija estaba en mi casa, sola (...) Sabía que profesionalmente estaba progresando, pero no era lo que quería, no, no, no me hacía plena, no me sentía plena (Belén, 40 años, 2 hijas/os).

Una jornada laboral que se asemeje al horario escolar, flexibilidad en los horarios, responsabilidades laborales que se limitan al tiempo de trabajo, son factores que ayudan a definir una conciliación como satisfactoria. Las entrevistadas que tienen hijas/os pequeñas/os, no desean disminuir el tiempo que comparten con ellos/as y rechazan -sin mostrarse afectadas- los puestos de trabajo que implican la implementación de un modelo androcéntrico de profesional, debido al costo familiar que este perfil supone. Para poder llevar a cabo un ejercicio deseado de la maternidad, suelen eliminar de sus posibilidades profesionales aquellos empleos más competitivos, los cuales vienen acompañados de mayor prestigio y remuneración.

Aunque se observa en muchos casos un rechazo a la figura del ama de casa-imagen que funciona como lo "otro" opuesto, necesario en la construcción de la identidad- las tareas que giraron tradicionalmente en torno de este rol permanecen. Las profesionales continúan identificándose como las principales responsables del trabajo afectivo. Entonces, si bien las entrevistadas son mujeres con profesiones socialmente valoradas y con percepciones sobre su trabajo muy positivas, siguen identificándose generalmente en su rol de cuidadoras, mientras que los varones profesionales se afirman en su rol de proveedores.

Desde esta perspectiva, en la construcción de las identidades profesionales femeninas se observa un proceso similar al que se conoce como "revolución estancada" (Hochschild y Machung, 1989; Wainerman, 2007). Así como el trabajo remunerado ha incrementado su importancia en relación a los proyectos de vida de las mujeres, las tareas domésticas y de cuidados no han perdido relativamente el mismo compromiso en la vida de las entrevistadas. De esta manera, aun cuando la esfera laboral funciona como "ámbito fundamental de sentido, acción y responsabilidad para las mujeres, no reemplaza ni se opone al espacio familiar, sino que es permanentemente articulado con éste" (Díaz, Godoy y Stecher, 2005, p. 58).

Entonces, la principal obligación que asumen las mujeres se sigue relacionando con el cuidado de sus hijos/ as. Es interesante observar la persistencia de un ideal de maternidad "intensiva". Este concepto define a la madre a partir de su gran dedicación a sus hijos/as en términos de tiempo, afecto y educación, asumiendo que es ella la que debe desarrollar el papel de cuidadora. Aunque la mayoría de las entrevistadas recurre -o lo hizo- a otras personas e instituciones de cuidado para poder desempeñar su trabajo, esto se describe muchas veces como una situación angustiante:

Si bien tenía una niñera que era re de confianza, era bastante doloroso para mí dejarlo, a veces se despertaba y lloraba que no quería que... Estuve a punto de renunciar, porque lloraba que no quería que me vaya a trabajar, que quería que me quede con él (Julieta, 39 años, 1 hijo)

Pese a que lloraba por los momentos que no estaban y los extrañaba. Consideré que [trabajar] era el mejor ejemplo que les podía dar. Y que en el tiempo y a esta edad, que de hecho ya lo puedo demostrar, los diálogos son muy diferentes que si yo no hubiera hecho nada más que ser ama de casa (Patricia, 58 años, 2 hijas/os).

La fuerte legitimidad de la crianza a tiempo completo, trae como consecuencia la supeditación de la trayectoria profesional a la esfera doméstica, por lo menos en los primeros años de cuidado. En simultáneo al crecimiento de sus hijas/os, ellas pueden asumir mayores responsabilidades en el ámbito laboral. Esta situación genera, por un lado, que su condición de profesionales no les permita ejercer la maternidad que desearían -en cuestiones de tiempo- lo que les provoca sentimientos de culpa. Por otro lado, que sacrifiquen algunos requerimientos profesionales para responsabilizarse de los deberes familiares. Esto desemboca en complicadas estrategias que perjudican su calidad de vida (Solé y Parella, 2008). En otras mujeres, como se mencionó anteriormente, este dilema se resuelve postergando su maternidad a un punto de su carrera laboral 
en el cual los costos no sean tan elevados. En este sentido, Mabel Burín (2011) utiliza el concepto de la "opción de hierro" para referirse a la falta de libertad de elección en una determinada situación. Esto queda particularmente reflejado en la dicotomía entre desarrollar una carrera laboral o la crianza y atención familiar satisfactorias.

\section{Consideraciones Finales}

El campo de la ciencia y la tecnología, y particularmente la ingeniería, siguen constituyéndose como espacios fuertemente masculinizados. No solo por la alta tasa de participación masculina, sino que también se mantiene la identificación de este ámbito como un asunto de varones. Como ya se mencionó, alrededor de la ingeniería se erigieron verdaderas fronteras en torno al género. Para atravesarlas y pertenecer a ese territorio, las mujeres sufren un proceso de aculturación. Esta situación nos invita a suponer que una parte de las estudiantes de ingeniería pueden haber sido expulsadas antes de lograr insertarse en la profesión. Asimismo, aunque la aculturación implique la renuncia a parte de la propia identidad, la adaptación no significó para la mayoría de las entrevistadas la incorporación de un modelo androcéntrico de profesional, sino que -como se pudo observar en el segundo apartado- se reforzó su identidad femenina, funcionando como factor de valorización de sus trayectorias.

En este sentido, las mujeres construyen una identidad de género dentro del espacio profesional, manteniendo algunas de las aptitudes adquiridas en su socialización temprana y diferenciada. Defienden nuevos perfiles profesionales caracterizados por la valorización del respeto y la comprensión, en detraimiento del papel de la autoridad. Por lo tanto, la construcción de las identidades profesionales se encuentra interrelacionada con las características de género tradicionales, dando paso a nuevas formas de ejercer la profesión. Esto no impide que algunas de las entrevistadas acepten los estereotipos impuestos y se perciban incapaces de ejercer determinados puestos de trabajo, o acepten tareas muy poco relacionadas con su profesión.

Para lograr una mayor igualdad, deberíamos comenzar a desarticular la ciencia y la tecnología con el género masculino. Para esto, es interesante observar las identidades y perfiles profesionales que desarrollan las ingenieras. Ahora bien, aunque un gran número de estas mujeres valorizan las habilidades consideradas femeninas para ejercer su profesión, ¿lo hacen de la misma manera sus empleadores?, ¿qué consecuencias trae para su desarrollo profesional mantener este perfil? Como se afirmó anteriormente, no todas le otorgan esta valoración positiva a su género. Algunas de estas mujeres aceptan tareas para las que están sobrecalificadas o incorporan estereotipos que perjudican su ascenso laboral. Retomando a Sara Rietti y Diana Maffia (2005) la ciencia -y también la tecnología- lleva implícito un modelo de sujeto que se maneja naturalmente en la objetividad. Esto, en la organización binaria en la que se ha fundado lo social, corresponde a un sujeto masculino. Aceptar nuevas formas de acercarse al conocimiento -y a la producción tecnológica- significa no dejar de lado lo intrínsecamente humano que quedó fuera del quehacer científico, como las emociones, los afectos, los intereses o vínculos (Rietti y Maffia, 2005).

Gran parte de las entrevistadas se reconoce en este aspecto. Esto se puede ver en la valoración de su rol maternal y la legitimidad que le brindan a su principal responsabilidad como cuidadoras. La relación inversamente proporcional entre la identidad profesional y maternal, en donde a medida que se valoriza más un rol disminuye el otro, nos muestra que la conciliación entre ambas esferas es aún dificultosa. Aunque estas mujeres están en condiciones privilegiadas con respecto a otras trabajadoras, se mantiene la desigualdad en las responsabilidades de cuidado por el ideal de maternidad vigente. Es significativo observar cómo, aún en las mujeres altamente instruidas -lo que implica importantes cantidades de tiempo invertidas en elloy con gran capacidad de desarrollo profesional, se defiende un tipo de maternidad intensiva, en la cual las responsabilidades en torno a la crianza y las tareas dentro del hogar permanecen y obstaculizan sus progresos profesionales. 
Para terminar, es pertinente señalar que, más allá de las barreras objetivas, existen aspectos subjetivos relacionados a las identidades de género que dificultan el camino hacia una mayor igualdad. Para mejorar esta situación, es necesario comenzar por correr el foco del problema de las mujeres y problematizar el funcionamiento de la institución científico-tecnológica. Preguntas acerca de las normas y valores que rigen este ámbito, pueden esclarecer los motivos que expliquen por qué son pocas las mujeres que se desempeñan en este sector.

\section{REFERENCIAS}

Actis De Pasquale, E. y Lanari, M. E. (2015). El mercado laboral escenario de desigualdades persistentes. Las brechas abiertas de Argentina. En M. E. Lanari y C. Hasanbegovic (Eds.). Mujeres de Latinoamérica. El presente en veintidós letras (pp. 213-270). Recuperado de http://nulan.mdp.edu.ar/2403/

Arango, L. G. (2006). Género e ingeniería: la identidad profesional en discusión. Reflexiones a partir del caso de las ingenieras en Sistemas en la Universidad Nacional de Colombia. Revista Latinoamericana de Estudios del Trabajo, 12(18), 199-223. Recuperado de https://dialnet.unirioja.es/servlet/articulo?codigo $=2739308$

Barberá, E., Ramos, A. y Sarrió, M. (2003). Mujeres directivas, espacio de poder y relaciones de género. Anuario de Psicología, 34(2), 267-278. Recuperado de http://roderic.uv.es/handle/10550/2080

Barrère-Maurisson, M. A. (1999). La división familiar del trabajo. La vida doble. Buenos Aires: Asociación Trabajo y Sociedad Programa de Investigaciones económicas sobre tecnología, trabajo y empleo (CONICET), Lumen/ Hvmanitas.

Burín, M. (1996). Género y psicoanálisis: subjetividades femeninas vulnerables. En M. Burín y E. Dio (Eds). Género, psicoanálisis, subjetividad (pp. 61-99). Buenos Aires: Paidós.

Burín, M. (2011). Laberintos de cristal en la carrera laboral de las mujeres. El rol de las mentoras (s/d) Recuperado de http://www.oei.es/congresoctg/memoria/pdf/Mburin.pdf

Conway, J. K., Bourque, S. C. \& W. J., Scott (Eds.). (1987). Learning about Women: Gender, Politics and Power. The University of Michigan Press: Ann Arbor.

Crenshaw, K. (1995). The intersection of race and gender. En K. Crenshaw, et. al. (Ed.) Critical Race Theory (pp. 357-383). New York: The New Press.

Cuadrado, I. (2003). ¿Emplean hombres y mujeres diferentes estilos de liderazgo? Análisis de la influencia de los estilos de liderazgo en el acceso a los puestos de dirección. Revista de Psicología Social, 18(3). https://doi.org/10.1174 /021347403322470864

Cutuli, R. y Aspiazu, E. (2015) Las políticas de cuidado infantil en Argentina. Aportes para su clasificación y evaluación. En M. E. Lanari y C. Hasanbegovic (Eds.). Mujeres de Latinoamérica. Elpresente en veintidós letras (pp. 339-371). Recuperado de http://nulan.mdp.edu.ar/2407/1/cutuli-aspiazu-2015.pdf

Díaz, X., Godoy, L. y Stecher, A. (2005). Significados del trabajo, identidad y ciudadanía. La experiencia de hombres y mujeres en un mercado laboral flexible. Cuaderno de Investigación $N^{o} 3$. Recuperado de http://mastor.cl/blog/wp-content/uploads/2016/09/Stecher.-Significados-del-trabajo.-Identidad-y-ciudad ania.-Stecher-Godoy-Diaz.pdf

Díaz, X., Godoy, L. y Stecher, A. (2007). Trabajo e identidades: continuidades y rupturas en un contexto de flexibilización laboral. En Guadarrama R. y Torres José Luis (Coords.) Los significados del trabajo femenino en el mundo global: estereotipos, transacciones y rupturas. Cuadernos A. Temas de Innovación Social. España: Anthropos y Universidad Autónoma Metropolitana.

Estébanez, M. E. (Coord.). (2003). La participación de la mujer en el sistema de Ciencia y tecnología en Argentina. Documento de trabajo No 8. Montevideo: Proyecto GENTEC/Grupo REDES/UNESCO Oficina Regional Montevideo.

Estébanez, M. E. (2004). Proyecto Iberoamericano de Ciencia, Tecnología y Género. Reporte Iberoamericano. Recuperado de: http://www.comunicacion.amc.edu.mx/comunicacion/docs/Reporte_Final\%20GENTEC.pd $\mathrm{f}$ 
Expósito Molina, C. (2012). ¿Qué es eso de la interseccionalidad? Aproximación al tratamiento de la diversidad desde la perspectiva de género en España. Investigaciones Feministas, 3, 203-222. https://doi.org/10.5209/rev_INFE .2012.v3.41146

Fraser, N. (2015). Fortunas del feminismo. Madrid: Traficantes de sueños.

García Prince, E. (2008). Políticas de igualdad, equidad y gender mainstreaming. ¿De qué estamos hablando? Marco conceptual. España: Agència Catalana de Cooperació al Desenvolupament y Fondo Fiduciario España-PNUD. Recuperado de: http://www.americalatinagenera.org/es/documentos/doc_732_Politicasdeigualdad23junio08 .pdf

Guadarrama Olivera, R. (2008). Los significados del trabajo femenino en el mundo global. Propuesta para un debate desde el campo de la cultura y las identidades laborales. Estudios Sociológicos, 26(77) 321-342. Recuperado de: h ttps://www.redalyc.org/pdf/598/59826203.pdf

Heller, L. (2011). Mujeres en la cumbre corporativa: el caso de la Argentina. Revista del Centro de Estudios de Sociología del Trabajo (CESOT), 3, 69-96. Recuperado de: http://ojs.econ.uba.ar/index.php/CESOT/article/view/514

Hochschild, A. y Machung, A. (1989). The second shift. Working parents and the revoution at home. New York: Viking.

Instituto Nacional de Estadística y Censos (INDEC). (2020). Mercado de trabajo. Tasas e indicadores socioeconómicos (EPH). Segundo Trimestre 2020 (Informe No 174). Recuperado de: https://www.indec.gob. ar/uploads/informesdeprensa/mercado_trabajo_eph_2trim20929E519161.pdf

Kaufmann, A. E. (1996). Tercer milenio y liderazgo femenino. En L., Nuño Gomez (coord.). Mujeres: de lo privado a lo público (pp. 177-186). Madrid: Técnos.

Keller, E. F. (2001). Reflexiones sobre género y ciencia (fragmento). Asparkia. Investigació feminista, 12, 149-153. Recuperado de http://www.e-revistes.uji.es/index.php/asparkia/article/view/891

Kruk, C., Trinchin, R., de Mello, S., Vélez-Rubio, G. y Cantieri, R. (2020). Mujeres Con-Ciencia: una mirada a las Geociencias en Uruguay. Descentrada, 4(2). https://doi.org/10.24215/25457284e122

Lisbona, A., Sainz, M. y López-Sáez, M. (2004). Mujeres ingenieras: percepciones sobre su vida profesional. Revista de psicologia general y aplicada, 57(2), 161-180.

Loden, M. (1986). Feminine leadership, or how to succeed in business without one of the boys. New York: Time Books

López-Bassols, V., Grazzi, M., Guillard, C. y Salazar, M. (2018). Las brechas de género en ciencia, tecnología e innovación en América Latina y el Caribe. Banco Interamericano de Desarrollo. http://dx.doi.org/10.18235/0 001082

Lorenzo, M. F. (2016). Que sepa coser, que sepa bordar, que sepa abrir la puerta para ir a la Universidad: las académicas de la Universidad de Buenos Aires en la primera mitad del siglo XX. Ciudad Autónoma de Buenos Aires: Eudeba.

Munévar, D. I. (2011). Solvencia de las mujeres en los estudios de ciencia, tecnología y género. En L. G. Arango y M. V. Vigoya (Eds.). El género: una categoría útil para las ciencias sociales (pp. 229-258). Bogotá: Universidad Nacional de Colombia.

Palermo, A. I. (2012). Estrategias y proyectos profesionales de las estudiantes de carreras «masculinas». Prácticas y Discursos. Cuadernos de Ciencias Sociales 1. http://dx.doi.org/10.30972/dpd.11773

Panaia, M. (2013). La profesión de ingeniero y el largo camino recorrido por las mujeres ingenieras. Trabajo presentado en VII Congreso Latinoamericano de Estudios de Trabajo, São Paulo, Brasil.

Rietti, S. y Maffia, D. (2005). Género, ciencia y ciudadanía. Arbor. Ciencia, pensamiento y cultura, 181(716), 539-544.

Rubin, G. (1986). El tráfico de mujeres. Notas para una 'economía política' del sexo. Nueva Antropología, 7(30), 95-145. Recuperado de: https://www.redalyc.org/pdf/159/15903007.pdf

Rubio Mayoral, J. L. (2006). Desarrollo económico y educación. Indicios históricos en las primeras "revoluciones industriales". Educación XXI, 9(1), 35-55. https://doi.org/10.5944/educxx1.9.0.318

Secretaria de Políticas Universitarias de la Nación (SPU). (s.f.). Sistema de consulta estadísticas universitarias. Recuperado a partir de: http://estadisticasuniversitarias.me.gov.ar/\#/home 
Secretaria de Políticas Universitarias de la Nación (SPU). (s.f.a). Anuarios estadísticos. Ministerio de Educación. Recuperado a partir de: https://www.argentina.gob.ar/educacion/universidades/informacion/publicaciones/a nuarios

Solé, C. y Parella, S. (2008). "Nuevas" expresiones de la maternidad. Las madres con carreras profesionales "exitosas". Revista Española de Sociología, 4, 67-92. Recuperado de https://recyt.fecyt.es/index.php/res/article/view/6494

Wainerman, C. H. (2007). Mujeres que trabajan. Hechos e ideas. En S. Torrado (Ed.), Población y bienestar en la Argentina del primero alsegundo centenario. Una bistoria social del siglo XX, Tomo II (pp. 325-352). Buenos Aires: Edhasa.

Wajcman, J. (2006). El tecnofeminismo. Valencia: Ediciones Cátedra, Universitat de Valencia, Instituto de la Mujer.

\section{Notas}

1 Las entrevistas fueron elaboradas por mi autoría, durante agosto y noviembre de 2016. Considerando que las lecturas y sentidos que se le otorgan a las trayectorias de vida propia no varían significativamente en cortos períodos de tiempo, encuentro que este material de análisis se mantiene vigente. Sus nombres fueron cambiados para preservar su identidad.

2 Ver SPU (s.f.), consultado el día 14/10/2020.

3 McGyver es un agente secreto que protagoniza la serie que lleva su nombre, creada en 1985 por una productora norteamericana. Lo que caracteriza al personaje es su ingenio y sus conocimientos técnicos, que se ponen en juego cada capítulo al improvisar instrumentos que lo ayudan a sortear los peligros que se le presentan.

4 Para un estudio más detallado sobre las percepciones de la discriminación de las mujeres en ciencia, ver Kruk, et al (2020).

5 Ver SPU (s.f.a).

6 No se tienen en cuenta las entrevistadas que ejercían como docentes.

7 Otros elementos importantes están relacionados a las mayores responsabilidades domésticas que recaen en las mujeres. Esto, implica que se las prepare desde la infancia para este tipo de labor. Como consecuencia, se dificulta la apropiación de aquellas características consideradas tradicionalmente como masculinas, y beneficiosas al mundo laboral. Además, sufren mayores dificultades en el desarrollo laboral debido a la doble jornada y a las complicaciones en la conciliación entre la esfera familiar y laboral.

8 Aun sin conocer su historia de vida, es pertinente problematizar de qué manera las mujeres consiguen romper con el techo de cristal. Es común que adopten un modelo androcéntrico de profesional, el cual muchas veces les impide desarrollar una vida familiar acorde a sus deseos. Teniendo en cuenta las altas tasas de masculinidad entre los trabajadores de astillero, otras cuestiones que no se pueden obviar son las mayores exigencias y las discriminaciones que pudo haber sufrido dicha persona.

9 Según datos de la EPH, durante el primer trimestre del 2020, el 49,5\% de ellas tenían trabajo o se encontraban en su búsqueda. Si nos centramos en la franja etaria que va desde los 30 a los 64 años, este porcentaje asciende al 67,5\%. En Mar del Plata, estos números se elevan al 53,4\% y 77,9\% respectivamente (INDEC, 2020). 